Trauma Berufskrankh 2004 - 6 [Suppl 2] : S236-\$240 DOI 10.1007/s10039-003-0764-6

Online publiziert: 13. August 2003

(c) Springer-Verlag 2003

T. Mückley • G.O. Hofmann • V. Bühren • BG-Unfallklinik, Murnau

\title{
Weiterentwicklungen der Verriegelungsoptionen von intramedullären Implantaten
}

nagelosteosynthese mit dickeren, soliden Marknägeln, die Verriegelungsmöglichkeiten vorhielten.

\section{Materialeigenschaften}

Bei dem T2-Marknagelsystem handelt es sich um kanülierte Marknägel aus einer Titanlegierung (Ti6 $\mathrm{Al}_{4} \mathrm{~V}$ ) Anodisation Typ 2.

Vorteile

Vorteile der Implantate aus Titan sind u. a. die geringeren Bildstörungen in der bildgebenden Diagnostik mittels CT und MRT. Des Weiteren kann die zunehmende Allergieproblematik bei der Verwendung von Implantatstählen durch den Einsatz von Titanimplantaten umgangen werden. Bezüglich des Elastizitätsmoduls liegen Marknägel aus Titanlegierungen näher am physiologischen Elastizitätsmodul des Knochens als Stahlimplantate. Es gibt experimentelle Hinweise dafür, dass die Frakturheilung dadurch positiv beeinflusst werden kann [17].

Durch die Anodisationsverfahren konnten die Oberflächen der Titanimplantate verändert werden. Dadurch werden niedrigere Abriebs- und Verschleißraten erreicht, die mit denen von Stahlimplantaten vergleichbar sind. Bezüglich der Verriegelungsschrauben wirken sich diese Materialeigenschaften insofern positiv aus, dass für 5-mm-Verriegelungsschrauben aus Titanlegierung Anodisation Typ 2 vergleichbare Dauerfestigkeitswerte wie für 6,28 -mm-Verriegelungsschrauben aus Implantatstahl erzielt werden. Hinweise bezüglich einer geringeren Adhäsion von Bakterien auf Oberflächen von Titanimplantaten vs. Stahlimplantaten haben sich auf die Implantatewahl in der septischen Knochenchirurgie ausgewirkt [1].

\section{Nachteile}

Nachteile der Implantate aus Titanlegierungen sind

- die höheren Kosten,

- das stärkere Einwachsverhalten und

- die fehlenden Konturierungsmöglichkeiten.

\section{Endständige Verriegelungsoptionen}

Die Verriegelungsmöglichkeiten an der Nagelbasis und der Nagelspitze wurden ursprünglich zur Rotations- und Längensicherung des Repositionsergebnisses von Schaftfrakturen genutzt. In modernen Nagelsystemen sind die Verriegelungsmöglichkeiten mittlerweile weiter endständig angeordnet, um auch die Stabilisierungen von Schaftfrakturen im 
1. und 5. Fünftel sowie von metaphysären Frakturen zu ermöglichen (• Abb. 1). Des Weiteren können die Humerus- und Femurnägel in antegrader sowie retrograder Technik eingesetzt werden, wodurch solche Nagelsysteme als intramedulläre Universalimplantate ein möglichst großes Indikationsspektrum abdecken. Hiermit können die Vorhaltekosten gesenkt und die Übungshaltung mit dem Nagelsystem verbessert werden.

\section{Winkelstabilität}

Durch die Entwicklung von kurzen, axial in das Nagellumen einführbaren Kompressionsschrauben können eine Verblockung der Verriegelungsschrauben und somit eine angenäherte Winkelstabilität erzielt werden (• Abb. 2). Dies kann für spezielle Indikationen, wie z. B. proximale Tibiafrakturen oder Frakturen bei ausgeprägter Osteoporose, genutzt werden (• Abb. 3).

\section{Apposition}

Als Risikofaktoren für eine verzögerte Knochenbruchheilung oder Pseudarthrosenausbildung gelten neben Frakturen mit ausgeprägtem Weichteilschaden und Querfrakturen u. a. auch ein fehlender Fragmentkontakt und ein klaffender Frakturspalt [4]. Im Rahmen der Marknagelung mit dem T2-System kann über die Kompressionsschraube Schub auf eine proximale Verriegelungsschraube in einem Längsloch ausgeübt werden (• Abb. 4). Hierdurch kann eine möglichst gute Aneinanderlagerung der Fragmentenden erzielt werden, und ein klaffender Frakturspalt wird vermieden (• Abb. 5) [11]. Diese integrierte Appositionsmöglichkeit wird in allen T2-Nageltypen durch ein Gewinde im Nagellumen an der Nagelbasis und ein in diesem Bereich angeordnetes Längsloch gewährleistet. Wird keine Apposition der Fraktur benötigt, kann das Längsloch zumindest für eine dynamische Verriegelungsmarknagelung genutzt werden.

\section{Kompressionsmarknagelung}

Viele der konventionellen Verriegelungsmarknagelsysteme benötigen die Markraumaufbohrung, um mit größervolumi-

Trauma Berufskrankh 2004 - 6 [Suppl 2] : S236-S240

DOI 10.1007/s10039-003-0764-6

C Springer-Verlag 2003

T. Mückley · G.O. Hofmann · V. Bühren

\section{Weiterentwicklungen der Verriegelungsoptionen von intramedullären Implantaten}

\section{Zusammenfassung}

Mit modernen ausgereiften Marknagelsystemen, wie dem T2-System, kann ein breites Indikationsspektrum abgedeckt werden. Neben Frakturen, Pseudarthrosen und Osteotomien im Schaftbereich können durch endständig angeordnete Verriegelungsoptionen auch metaphysäre Frakturen und Pseudarthrosen stabilisiert werden. Die Materialeigenschaften der Titanlegierung Anodisation Typ II konnten bezüglich niedrigerer Abriebs- und Verschleißraten und eines physiologischeren Elastizitätsmoduls optimiert werden. Die Entwicklung von kurzen Kompressionsschrau-

ben, welche axial in das Nagellumen eingeführt werden können, ermöglicht eine Verblockung der Verriegelungsschrauben im Bereich der Nagelbasis und somit eine angenäherte Winkelstabilität. Durch diese Kompressionsschraube können auch eine feindosierte Aneinanderlagerung der Hauptfragmente und ggf.auch Kompression auf die Fraktur bzw. Osteotomie ausgeübt werden.

\section{Schlüsselwörter}

Verriegelungsoptionen · Kompressionsmarknagelung · Apposition · Winkelstabilität

\section{Further advances in locking options for intramedullary implants}

\section{Abstract}

Modern, well-developed medullary nailing systems such as the $T 2$ system can be used for a wide range of indications. In addition to fractures, malunions and osteotomies in the region of the shaft, locking systems are already configured for use in metaphyseal fractures and malunions. The material properties of the type II anodised titanium alloy are now optimized insofar as friction and wear rates are reduced and its mechanical properties are more physiological.The development of short compression screws that can be introduced axially into the nail makes it possible to block the locking screws in the region of the nail base and thus to achieve approximately fixed angles. This compression screw also allows finely tuned apposition of the main fragments and, if appropriate, to apply compression on the fracture or osteotomy.

\section{Keywords}

Locking options · Compression nailing

Apposition · Angular stability 


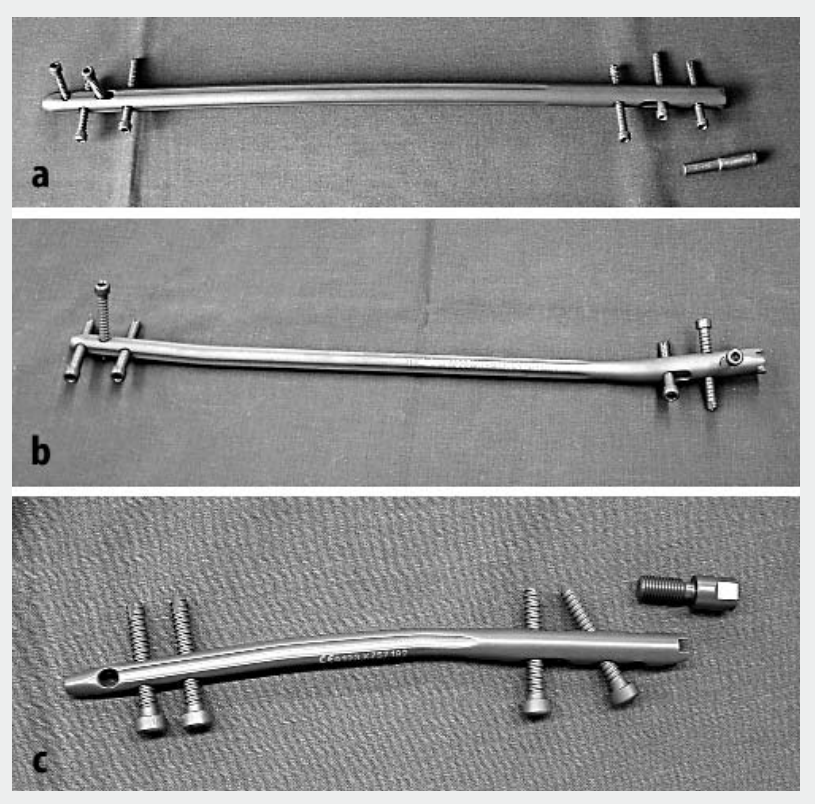

Abb. $1<$ Endständige Verriegelungsoptionen an den distalen und proximalen Nagelenden für die Produktreihen Femur, Tibia und Humerus (von oben nach unten)

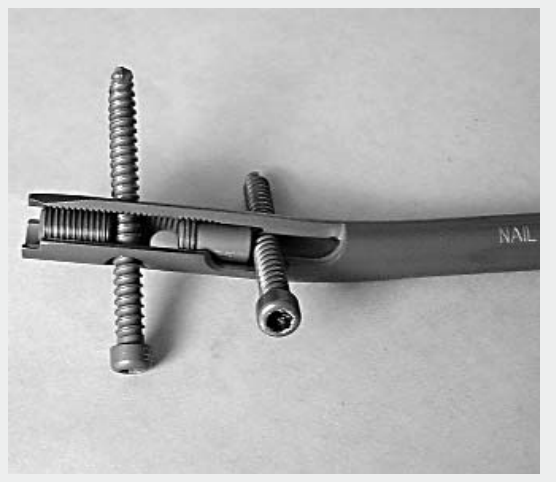

Abb. $2<$ In das Nagellumen einführbare kurze Kompressionsschrauben zur Verblockung der Verriegelungsschrauben (angenäherte Winkelstabilität) gen Implantaten eine ausreichende biomechanische Stabilität zu gewährleisten. Es gibt experimentelle und klinische Daten, die Hinweise geben, dass der Aufbohrvorgang nachteilige Auswirkungen, wie

- systemische Embolisation,

- Beeinträchtigung der Lungenfunktion und Blutgerinnung,

- Reduktion der Knochensteifigkeit und

- Beeinträchtigung der endostalen Blutversorgung

haben kann $[8,13,15,16,19]$.

Entsprechend dieser möglichen nachteiligen Auswirkungen geht der Trend der Marknagelosteosynthese von Schaftfrakturen hin zu limitiert-aufgebohrten und unaufgebohrten Techniken.
Für die unaufgebohrten Marknageltechniken mit schmalkalibrigen Nägeln und dünnen Verriegelungsbolzen wurde über eine erhöhte Inzidenz von Komplikationen, wie

- Implantatversagen,

- Fehlheilungen und

- Störungen der Knochenbruchheilung

berichtet $[6,9]$. Viele der angesprochenen Komplikationen können auf eine initial nicht ausreichende Stabilität der Osteosynthese zurückgeführt werden [6]. Klinisch kann sich dies durch prolongierte Schmerzzustände und Verzögerungen im Belastungsaufbau darstellen.

Eine klare Verbesserung hinsichtlich dieser biomechanisch nicht ausreichenden Eigenschaften stellt die Entwicklung von Verriegelungsmarknägeln mit integrierter Kompressionsmöglichkeit dar. Durch die komprimierbare intramedulläre Osteosynthese kann eine verbesserte Primärstabilität, verglichen mit anderen nichtkomprimierten intramedullären Osteosynthesen, erzielt werden $[2,3,7,14]$. Ebenso kann durch den Kompressionsmechanismus, welcher eine kontrollierte Apposition der Fragmente erlaubt, eine Diastase im Frakturspalt vermieden werden $[3,12]$.

\section{Indikationen der Kompressionsmarknagelung}

Die Indikation zur Kompressionsmarknagelung ergibt sich aus der axialen Stabilität der vorliegenden Fraktur oder Osteotomie: Einfache Frakturen, wie Quer- und Schrägfrakturen, Pseudarthrosen und quere Osteotomien, sind ideale Indikationen [3].

Von besonderem Wert ist die Kompressionsmarknagelung in Situationen, in denen die Autokompression durch die Belastung der Extremität nicht möglich ist. Hierzu zählen Frakturen und Pseudarthrosen des Humerusschafts und Kettenverletzungen der unteren Extremität, die eine Teilbelastung der Extremität nicht erlauben [3].

\section{Biomechanisches Prinzip der Kompressionsmarknagelung}

Das biomechanische Prinzip der Kompressionsmarknagelung beruht auf der Anwendung eines intramedullären Implantats, das unverklemmt in die Markhöhle eingebracht wird und nach der Verriegelung eine Relativbewegung der Fragmente gegeneinander ermöglicht. Dies gelingt durch einen Verriegelungsbolzen, welcher in einem Langloch das dem Nageleintrittspunkt entsprechende Hauptfragment so fixiert, dass eine axiale Relativbewegung zwischen Nagel und Schraube noch möglich ist [8].

Mit einer axial eingebrachten Schubschraube wird über ein Maschinengewinde Schub auf den Verriegelungsbolzen und damit auf das proximale Hauptfragment ausgeübt. Dieser Schub führt zu einer Relativbewegung des proximalen Fragments über den Marknagel bzw. des 

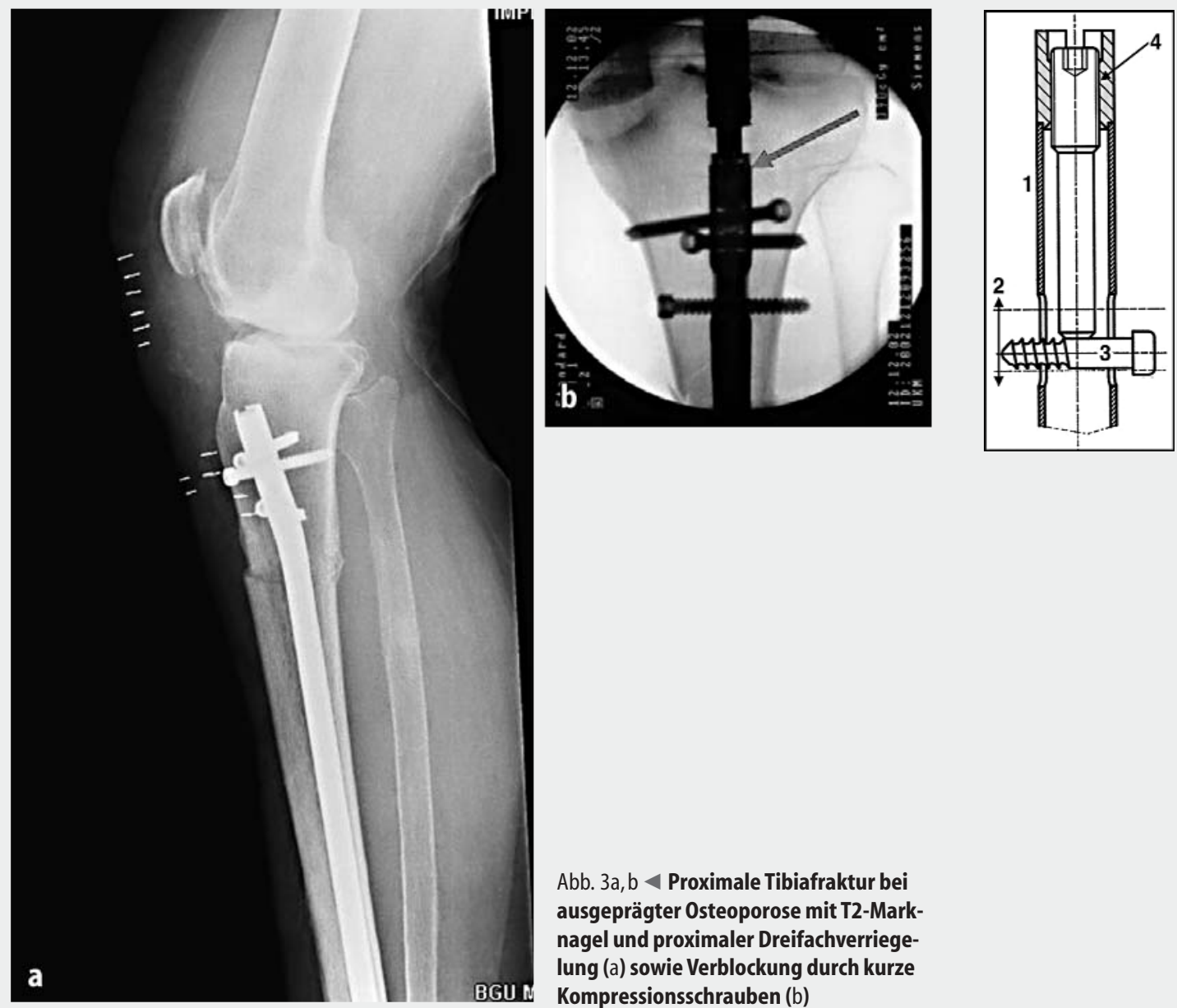

Abb. $4<$ Schematische Darstellung der Nagelbasis mit integriertem Kompressions- bzw. Appositionsmechanismus: kanülierte Nagelbasis mit Innengewinde (1), längsovales Verriegelungsloch (2), Verriegelungsschraube (3), in das Nagellumen eingeführte Kompressionsschraube (4)

Abb. $3 a, b<$ Proximale Tibiafraktur bei ausgeprägter Osteoporose mit T2-Marknagel und proximaler Dreifachverriegelung (a) sowie Verblockung durch kurze Kompressionsschrauben (b)
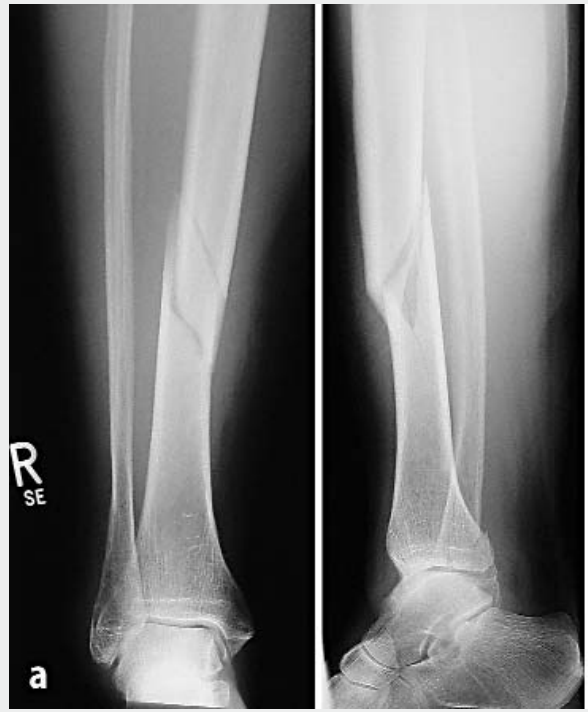
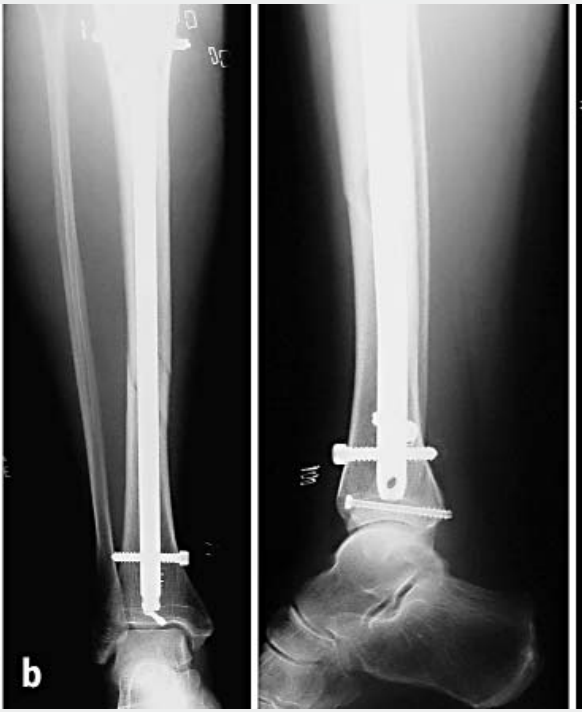

Abb. 5a, b $<$ Tibiaschaftspiralfraktur mit Fraktur des Volkmann-Dreiecks (a). Nach gedeckter Verschraubung des Volkmann-Dreiecks geschlossene Marknagelung und Apposition der Spiralfraktur über eine kurze Kompressionsschraube mit nachfolgender statischer Verriegelung (b) mit Verriegelungsschrauben fixierten distalen Fragments nach proximal, wodurch eine Annäherung und Kompression zwischen den Fragmenten aufgebaut wird [3].

Durch die Kompressionsmarknagelung kann eine wesentliche Zunahme der
Primärstabilität der Osteosynthese im Vergleich zu nicht komprimierten Marknagelosteosynthesen erreicht werden. Richardson et al. [14] konnten dies durch ihre biomechanischen Untersuchungen von verschiedenen intramedullären Os- teosynthesen des Femurs bestätigen. Im Vergleich zu einer nicht komprimierten Marknagelosteosynthese konnten Blum et al. [2] eine erhöhte Steifigkeit im 4Punkte-Biegetest sowie eine signifikant erhöhte Torsionssteifigkeit nachweisen. 
Ähnliche Ergebnisse konnten von anderen Autoren klinisch und experimentell bestätigt werden $[7,10,12]$.

Die gute Aneinanderlagerung und Aneinanderpressung der Fragmentenden im Frakturspalt führt neben einer erhöhten biomechanischen Primärstabilität zu einem Load-sharing des Knochen-Implantat-Verbunds. In der Folge wird bei Belastung der betroffenen Gliedmaße mehr der Knochen und weniger das intramedulläre Implantat beansprucht. Da es sich entsprechend den Indikationen zur Kompressionsmarknagelung um axial stabile Frakturen und Osteotomien handelt, kann die Osteosynthese mittels Kompressionsmarknagel in der Regel sofort beschwerdeorientiert belastet werden. Da es sich um eine dynamische Marknagelosteosynthese handelt, ist eine später geplante Dynamisierung, wie bei einer statischen unaufgebohrten Verriegelungsmarknagelung, prinzipiell nicht notwendig. Fraglich bleibt, über welchen Zeitraum hinweg die Kompression in vivo erhalten bleibt. Erfahrungen aus dem klinischen Alltag deuten darauf hin, dass es sich hier in den meisten Fällen um einen Zeitraum von etwa 2-4 Wochen handelt. Gerade in diesem Zeitraum kann eine erhöhte Stabilität durch die kontinuierliche Kompression des Frakturspalts, wie in tierexperimentellen Arbeiten gezeigt werden konnte, die Neuausbildung von Gefäßen im Bereich des Frakturspalts und somit die Frakturheilung positiv beeinflussen [18]

\section{Fazit}

Mit modernen ausgereiften Marknagelsystemen kann ein breites Indikationsspektrum abgedeckt werden. Neben dem Schaftbereich von Tibia, Humerus und Femur kann auch der metaphysäre Bereich durch endständig angeordnete Verriegelungslöcher stabilisiert werden. An Humerus und Femur kann zwischen einer antegraden bzw. retrograden Implantationstechnik gewählt werden. Dadurch werden die Anzahl der benötigten verschiedenen Nagelsysteme pro Klinik reduziert und die Vorhaltekosten gesenkt. Die Übungshaltung des instrumentierenden Personals und der Operateure wird verbessert.

Die Nagelsysteme werden mittlerweile zunehmend aus Titanlegierungen hergestellt. Mit der Titanlegierung Anodisation Typ II konnten die Oberflächeneigenschaften bezüglich niedrigerer Abriebs- und Verschleißraten verbessert werden. Das Elastizitätsmodul der Marknägel aus Titanlegierungen kommt dem von Knochen näher als Stahlimplantate.

Die Entwicklung von kurzen Kompressionsschrauben, welche axial in das Nagellumen eingeführt werden können, ermöglicht eine Verblockung der Verriegelungsschrauben im Bereich der Nagelbasis und somit eine angenäherte Winkelstabilität. Diese Kompressionsschraube ermöglicht des Weiteren eine feindosierte Aneinanderlagerung der Hauptfragmente und ggf. eine Kompressionsausübung auf die Fraktur bzw. Osteotomie.

\section{Korrespondierender Autor Dr. T. Mückley}

BG-Unfallklinik,

Prof.-Küntscher-Straße 8, 82418 Murnau,

E-Mail:T.Mueckley@gmx.de

\section{Literatur}

1. Arens S, Schlegel U, Printzen G, Ziegler WJ, Perren SM, Hansis M (1996) Influence of materials for fixation implants on local infection. An experimental study of steel versus titanium DCP in rabbits.J Bone Joint Surg Br 78-B: 647-651

2. Blum J, Machemer H, Hogner M, Baumgart F, Schlegel U, Wahl D, Rommens PM (2000) Biomechanics of interlocked nailing in humeral shaft fractures. Comparison of 2 nail systems and the effect of interfragmentary compression with the unreamed humeral nail. Unfallchirurg 103: 183-190

3. Bühren V (2000) Intramedullary compression nailing of long tubular bones. Unfallchirurg 103: 708-720

4. Claes L, Augat P, Suger G, Wilke H-J (1997) Influence of size and stability of the osteotomy gap on the success of fracture healing.J Orthop Res 15: 577-584

5. Court-Brown CM (1991) An atlas of closed nailing of the tibia and femur.Deutscher Ärzteverlag, Köln

6. Duda GN, Mandruzzato F, Heller M, Goldhahn J, Moser R, Hehli M, Haas NP (2001) Mechanical boundary conditions of fracture healing: borderline indications in the treatment of unreamed tibial nailing.J Biomech 34: 639-650

7. Gonschorek O, Hofmann GO, Bühren V (1998) Interlocking compression nailing: a report on 402 applications. Arch Orthop Trauma Surg 117: 433-437

8. Heim D, Regazzoni P, Tsakiris DA, Aebi T, Schlegel U, Marbet GA, Perren SM (1995) Intramedullary nailing and pulmonary embolism: does unreamed nailing prevent embolization? An in vivo study in rabbits.J Trauma 38: 899-906

9. Hutson J, Zych GA, Cole JD, Johnson KD, Ostermann P, Milne EL, Latta L (1995) Mechanical failures of intramedullary tibial nails applied without reaming. Clin Orthop 315: 129-137

10. Mittelmeier H, Trennheuser M, Mittelmeier W (1990) Vergleichende biomechanische Messungen der Torsionsstabilität von intramedullären Nagel-Osteosynthesen. Hefte Unfallheilkd 212: 468-471
11. Mückley T,Hofmann GO, Bühren V (2002) Weiterentwickelter Kompressions-Verriegelungs-Nagel. Biomaterialien 3: 38-41

12. Mückley T, Gonschorek O, Bühren V (2003) Compression nailing of long bones. Eur J Trauma 29: 113-128

13. Pratt DJ, Papagiannopoulos $G$, Rees PH, Quinnell R (1987) The effects of medullary reaming on the torsional strength of the femur. Injury 18: 177-179

14. Richardson T, Voor M, Seligson D (1998) Fracture site compression and motion with three types of intramedullary fixation of the femur. Osteosynthese Int 6: 261264

15. Schemitsch EH, Kowalski MJ, Swiontkowski MF, Senft D (1994) Cortical bone blood flow in reamed and unreamed locked intramedullary nailing: a fractured tibia model in sheep. J Orthop Trauma 8: 373-382

16. Schemitsch EH, Kowalski MJ, Swiontkowski MF, Harrington RM (1995) Comparison of the effect of reamed and unreamed locked intramedullary nailing on blood flow in the callus and strength of union following fracture of the sheep tibia.J Orthop Res 13: 382-389

17. Utvag SE, Reikeras $O$ (1998) Effects of nail rigidity on fracture healing. Strength and mineralisation in rat femoral bone. Arch Orthop Trauma Surg 118: 7-13

18. Wolf JW, White AA, Panjabi MM, Southwick WO (1981) Comparison of cyclic loading versus constant compression in the treatment of long-bone fractures in rabbits. J Bone Joint Surg Am 63-A: 805-810

19. Wozasek GE, Simon P, Redl H, Schlag G (1994) Intramedullary pressure changes and fat intravasation during intramedullary nailing: an experimental study in sheep. J Trauma 36: 202-207 